\title{
Registration and Tracking to Integrate X-Ray and MR Images in an XMR Facility
}

\author{
Kawal S. Rhode, Derek L. G. Hill*, Member, IEEE, Philip J. Edwards, John Hipwell, Daniel Rueckert, \\ Gerardo Sanchez-Ortiz, Sanjeet Hegde, Vithuran Rahunathan, and Reza Razavi
}

\begin{abstract}
We describe a registration and tracking technique to integrate cardiac $\mathrm{X}$-ray images and cardiac magnetic resonance (MR) images acquired from a combined X-ray and MR interventional suite (XMR). Optical tracking is used to determine the transformation matrices relating MR image coordinates and X-ray image coordinates. Calibration of $\mathrm{X}$-ray projection geometry and tracking of the $\mathrm{X}$-ray $\mathrm{C}$-arm and table enable three-dimensional (3-D) reconstruction of vessel centerlines and catheters from bi-plane X-ray views. We can, therefore, combine single X-ray projection images with registered projection MR images from a volume acquisition, and we can also display 3-D reconstructions of catheters within a 3-D or multi-slice MR volume. Registration errors were assessed using phantom experiments. Errors in the combined projection images (two-dimensional target registration error - TRE) were found to be 2.4 to $4.2 \mathrm{~mm}$, and the errors in the integrated volume representation (3-D TRE) were found to be 4.6 to $5.1 \mathrm{~mm}$. These errors are clinically acceptable for alignment of images of the great vessels and the chambers of the heart. Results are shown for two patients. The first involves overlay of a catheter used for invasive pressure measurements on an MR volume that provides anatomical context. The second involves overlay of invasive electrode catheters (including a basket catheter) on a tagged MR volume in order to relate electrophysiology to myocardial motion in a patient with an arrhythmia. Visual assessment of these results suggests the errors were of a similar magnitude to those obtained in the phantom measurements.
\end{abstract}

Index Terms-2-D-3-D registration, cardiovascular intervention, optical tracking, XMR systems.

\section{INTRODUCTION}

$\mathbf{X}$ -RAY AND MAGNETIC resonance (MR) interventional suites (XMRs) are a new type of facility for image-guided interventions, in which a cardiovascular X-ray set and magnetic resonance system are installed in the same interventional room. The configuration of the XMR system is generally of a MR scanner and an X-ray system positioned at opposite ends of the interventional room with a sliding patient table moving between the two. However, a system has been devised in which the X-ray system is integrated into the MR scanner [1], [2]. XMR facilities of the sliding table type are now available from all the major

Manuscript received January 31, 2003; revised July 29, 2003. This work was supported by the UK-EPSRC under Grant GR/R41019/1.

K. S. Rhode, P. J. Edwards, J. Hipwell, S. Hegde, V. Rahunathan, and R. Razavi are with the Division of Imaging Sciences, Guy's, King's \& St Thomas' School of Medicine, King's College London, Guy's Hospital, London SE1 9RT, U.K.

*D. L. G. Hill is with the Division of Imaging Sciences, Guy's, King's \& St Thomas' School of Medicine, King's College London, 5th Floor Thomas Guy House, Guy's Hospital, London SE1 9RT, U.K. (e-mail: derek.hill@kcl.ac.uk).

D. Rueckert and G. Sanchez-Ortiz are with the Department of Computing, Imperial College London, London SW7 2AZ, U.K.

Digital Object Identifier 10.1109/TMI.2003.819275
MR manufacturers, and are designed to facilitate the transition from X-ray guidance to MR guidance for endovascular procedures, in particular cardiac catheterization.

Cardiac catheterizations are undertaken either to obtain diagnostic information, or to perform image-guided interventions. Traditionally, cardiac catheters, guide wires and devices such as stents are visualised using single plane or bi-plane cine digital X-ray fluoroscopy. However, X-ray images have very poor soft tissue contrast so that the heart and blood vessels are not well visualised. Therefore, during the manipulation of catheters or devices, the operator has to rely on a mental knowledge of the anatomy obtained either from experience or previous X-ray contrast angiographic images.

MRI, on the other hand, provides much better soft tissue contrast, and the latest generation of scanners can acquire images at ten or more frames per second, providing the potential for MR image guidance in these procedures. However, as the great majority of the catheters, guide wires and devices used in cardiac catheterization procedures contain either ferromagnetic material or long electrical conductors, procedures on patients cannot currently be done under MR guidance alone. Hence, we have the concept of XMR hybrid systems. XMR enables parts of the procedure to be carried out under MR guidance, and parts under X-ray guidance. This will allow safe transition from X-ray-guided to MR-guided interventions, until such time as the devices are all MR compatible and MR visible, and the user-interface of the MRI systems is suitable for these interventions.

Initial evaluation of XMR technology demonstrated that this would be a promising technical solution to performing interventional MR procedures [3]. More recent work using animal models has shown that procedures such as stent placement and atrial septal defect closure can be carried out successfully in the XMR environment [4]-[6]. The clinical use of XMR has been demonstrated for interventional vascular procedures in the liver [7] and our group is currently undertaking a clinical programme of XMR guided cardiac catheterizations.

A limitation of the current generation of XMR technology is that, while the X-ray and MR systems are in the same room, and a patient can be moved easily between the two imaging devices, there is no capability for registration of the images. In this paper we show how registration and tracking technology, including techniques derived from those used in image-guided neurosurgery, can be used to provide registered X-ray and MR images during interventions. There are two important potential benefits to this approach. First, it would be possible to acquire X-ray images with overlays of previously acquired MR 


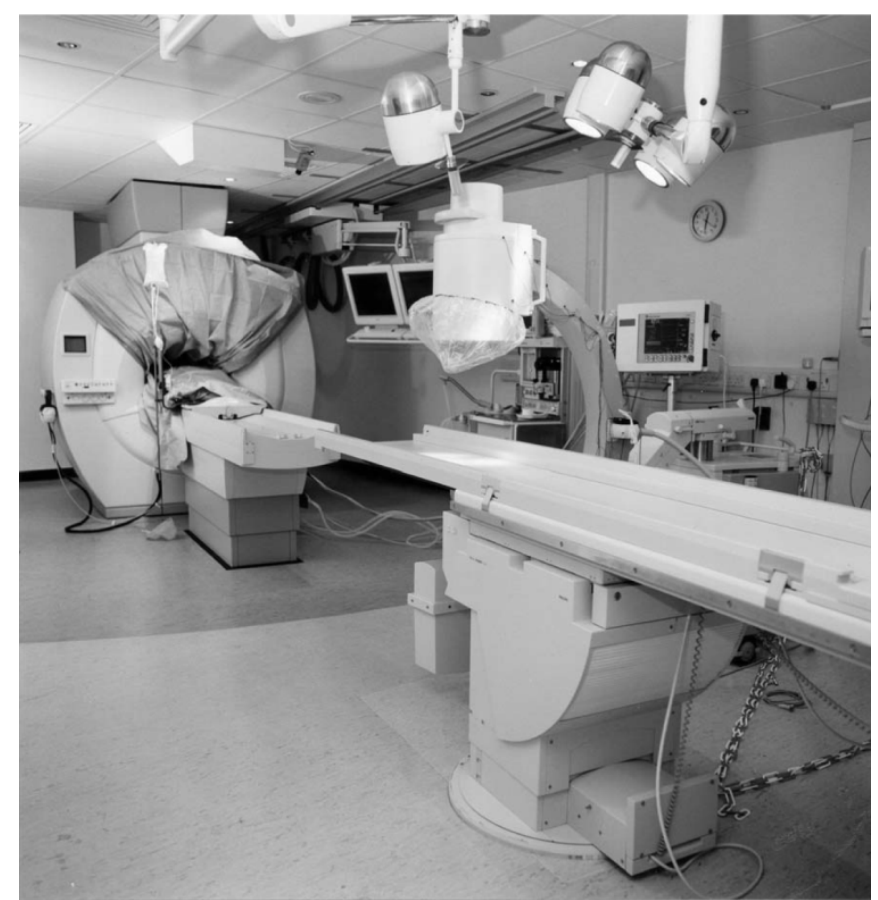

Fig. 1. XMR suite at Guy's Hospital, London, U.K. The change in the floor color indicates the transition from the MRI zone to the non-MRI zone.

images, so that the anatomical context can be provided by MR and the real time device tracking from X-ray. Second, it would be possible to generate the three-dimensional (3-D) locations of devices seen under X-ray guidance (rather than just projections) in MR coordinates using the information from the calibration and tracking. For example, it would be possible to carry out image-guided cardiac radio frequency (RF) ablation for the treatment of arrhythmias, in which the 3-D location of measurement electrodes used under X-ray guidance could be related to the 3-D location of functionally abnormal myocardium localized in MR.

\section{METHOD}

\section{A. Description of XMR Facility}

The XMR interventional suite (Fig. 1) at King's College London (Guy's Hospital Campus) comprises an X-ray and RF shielded room, with positive pressure air handling for sterility. The room contains a 1.5-T cylindrical bore MR scanner (Philips Intera I/T) and a mobile cardiac X-ray set (Philips BV Pulsera). The patient can be easily moved between the two systems using a specially modified sliding MR table top that docks with and transfers patients to a specially modified X-ray table (Philips Angio Diagnost 5 Syncratilt table). The room has two distinct zones: the MRI zone, in which the magnetic field is above 0.5 $\mathrm{mT}$, and the non-MRI zone, which comprises the rest of the room. The X-ray system is in the non-MRI zone, and the image intensifier is shielded with mu metal (nickel-iron alloy used for magnetic screening) to minimize MR-induced distortion. Patient transfer involves translation of the table top from the MR scanner to the X-ray table in the non-MRI zone. The docking and transfer takes less than $60 \mathrm{~s}$. An MR-compatible anaes-

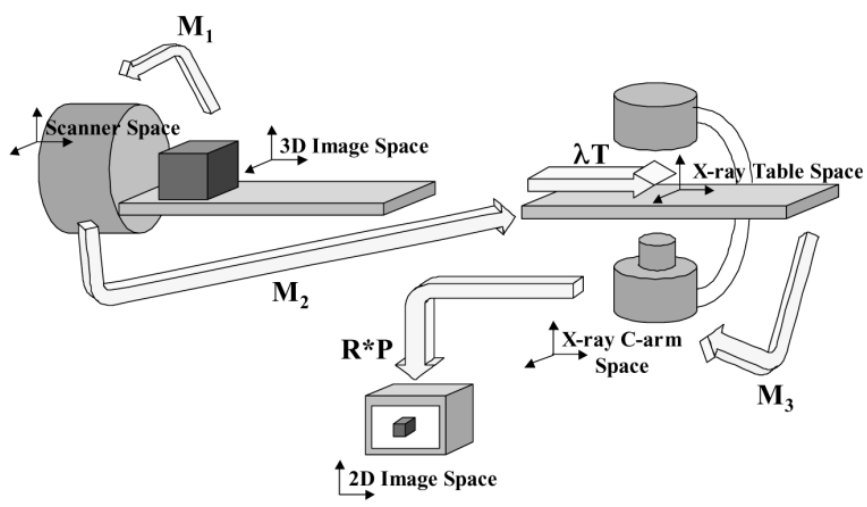

Fig. 2. Relationship between coordinate systems and transformation matrices.

thetic machine and equipment for monitoring ECG, peripheral oxygen saturation, inspired and expired $\mathrm{CO}_{2}$, anaesthetic gases, and invasive pressure are also incorporated. All connections to the anaesthetic and monitoring equipment are lengthened and secured to the sliding table top to allow them to remain in place during patient transfer.

The scanner can be controlled from inside the room using an MR-compatible console inside the MRI zone. This console can be used to adjust scan parameters such as slice thickness and orientation in real-time during imaging with the "interactive" sequence (SSFP sequence, $128 \times 128$ matrix, single slice, resolution $=2.5 \times 2.5 \mathrm{~mm} \times 8.0 \mathrm{~mm}, \mathrm{TR}=2.5 \mathrm{~ms}, \mathrm{TE}=1.25 \mathrm{~ms}$, and flip angle $=50^{\circ}$ ).

\section{B. Modifications for Tracking and Registration}

The XMR facility enables both X-ray and MR systems to be used during a single intervention, but does not provide the capability for registration of the images.

To register the images it is required to find the transformation that maps 3-D points in MR image space to two-dimensional (2-D) points in X-ray image space. This is achieved by a combination of system calibration and tracking. It is necessary to track the moving components of the XMR system. These comprise the X-ray C-arm, the X-ray table, and the sliding MR table top. The table top is automatically tracked by the MR scanner when docked with the MR table. The X-ray C-arm and the X-ray table each have six infrared emitting diodes (IREDs) affixed, which are tracked by a Northern Digital Optotrak 3020 (NDI, Ontario, Canada) positioned in the non-MRI zone of the room.

We, therefore, have the following coordinate systems: MR image space, $\mathrm{X}$-ray image space, $\mathrm{X}$-ray table space, and X-ray $\mathrm{C}$-arm space. The relationship between these coordinate systems is shown in Fig. 2. The Optotrak is able to locate the IRED markers placed on the two X-ray components. The location of these markers is in Optotrak space. For the two X-ray components a rigid body transformation matrix is calculated that maps points from X-ray $\mathrm{C}$-arm space and X-ray table space to Optotrak space. Let $\mathbf{X}_{C}$ be the transformation from X-ray C-arm space to Optotrak space, and let $\mathbf{X}_{T}$ be the transformation from $\mathrm{X}$-ray table space to Optotrak space. Then

$$
\mathbf{M}_{3}=\mathbf{X}_{C}^{-1} \mathbf{X}_{T}
$$




\section{Calibration of X-Ray Perspective Geometry}

Conventional calibration of a projection-imaging device, such as an X-ray set, involves acquiring images with a calibration object containing at least six points of known 3-D location in the field of view. Marking the location of these points in the 2-D images and solving for the 11 unknown elements then determines the $3 \times 4$ perspective projection matrix.

In our application, however, the X-ray images are acquired from many different views, and the perspective projection will change with view due to nonrigidity of the X-ray gantry, and the effect of the earth's magnetic field and adjacent MR system on the image intensifier. We initially carried out conventional calibrations from multiple views to quantify the position dependence using an acrylic cube ( $6 \mathrm{~cm}$ across) with 14 steel markers. We then devised a novel technique to calculate an "average" projection matrix based on multiple views.

The perspective projection calibration process involves acquisition of multiple X-ray views of an acrylic calibration object that can accept 14 point markers. The markers are based on those used in the Acustar Neurosurgical guidance system [8], and our version has previously been described by Edwards et al. [9]. These markers have alternative caps for use in MR and $\mathrm{X}$-ray imaging (containing a mixture of gadolinium and iodine contrast material), X-ray imaging alone (containing 3-mm-diameter steel ball bearings), and physical caps for use with a pointing device that is tracked with the Optotrak. The calibration object needed to be imaged with both MR imaging and X-ray imaging without being moved with respect to the sliding table top. Therefore, it was placed in the head coil that was firmly fixed to the sliding table top. A bottle containing copper sulphate solution was also placed within the head coil to give sufficient loading. Initially the table top was docked to the X-ray table and the physical markers were placed on the calibration object. Only 10 out of the 14 markers were used because four were inaccessible in the head coil. The position of the markers was located in X-ray table space using the pointing device and the Optotrak. The positions were marked three times and averaged to reduce location error. These markers were then changed for the ball bearing markers. Twelve tracked X-ray images were acquired with the X-ray gantry being moved to cover the typical locations used for interventions [gantry angles $0^{\circ}, 30^{\circ}, 60^{\circ}$, and $90^{\circ}$ from AP to lateral and translating parallel to the X-ray table by 0.00 , 0.25 , and 0.50 meters $(\mathrm{m})]$. Although three electronic magnification settings are allowed by the X-ray system, our current system only supports the largest field of view size $(23 \mathrm{~cm})$, so the calibration images and the subsequent patient images must be acquired using the same magnification setting. Fig. 3 shows one of the calibration images. Not all markers were visible in all views, therefore, the user had to interactively label each marker. In total the position of 48 markers was found in 2-D by manual marking followed by a local center of gravity approach. The corresponding 3-D positions were calculated by transforming the previously determined marker positions in X-ray table space to $\mathrm{C}$-arm space using the matrix $\mathrm{M}_{3}$ for each $\mathrm{X}$-ray image. Now

$$
\mathbf{P C}_{3-\mathrm{D}}=\mathrm{C}_{2-\mathrm{D}}
$$

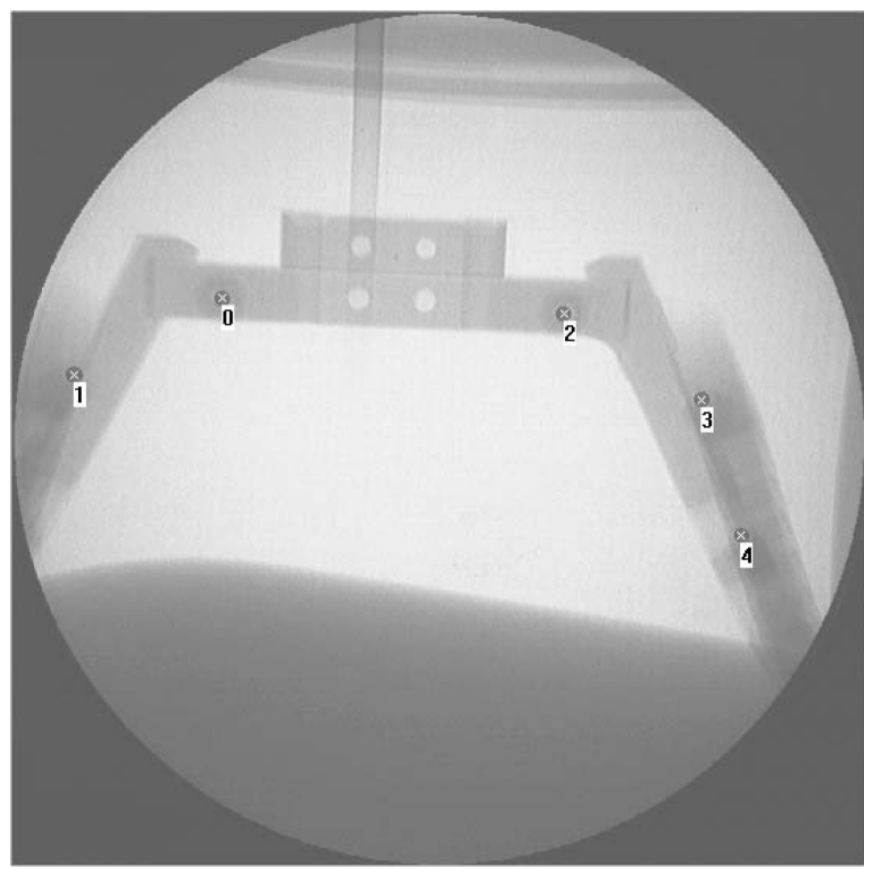

Fig. 3. X-ray view of the calibration object with ball bearing markers installed. The crosses show the location of the markers found manually.

where $\mathbf{C}_{3-\mathrm{D}}$ is the matrix of $3-\mathrm{D}$ point coordinates, $\mathbf{C}_{2-\mathrm{D}}$ is the matrix of corresponding 2-D point coordinates, and $\mathbf{P}$ is the perspective projection matrix. Equation (2) leads to an over determined set of linear equations and $\mathbf{P}$ was then found using singular value decomposition.

\section{Calibration of the Transformation Between 3-D Image Space and X-Ray Table Space}

As described above, the sliding table top is tracked by the Optotrak while docked with the X-ray table. The working volume of the Optotrak is too small to also track the table top in the MR scanner, and in any case the line of site would be obscured. This is not a problem, however, as the MR scanner software tracks the position of the table top while it is docked with the MR scanner. It is only necessary to carry out a calibration before the start of the procedure to determine the transformation between MR scanner space and the X-ray table space (matrix $\mathbf{M}_{2}$ ). In order to determine this transformation, the sliding table top is docked with the MR scanner with the calibration object still in place from the projection calibration stage. The ball bearing markers are replaced with MR imaging markers prior to this. The calibration object is then imaged using a T1-weighted volume MR scan $(256 \times 256$ matrix, 200 slices, resolution = $1.05 \mathrm{~mm} \times 1.05 \mathrm{~mm} \times 1.2 \mathrm{~mm}, \mathrm{TR}=14.0 \mathrm{~ms}, \mathrm{TE}=6.0 \mathrm{~ms}$, and flip angle $=15^{\circ}$ ). Fig. 4(a) shows surface renderings of the image data. The 3-D position of the markers is determined in MR image space using manual marking followed by a local center of gravity calculation. Now

$$
\mathbf{F}_{X R T}=\mathbf{M}_{2} \mathbf{M}_{1} \mathbf{F}_{I}
$$

where $\mathbf{F}_{X R T}$ is the matrix of marker locations in X-ray table space (determined earlier using the pointing device and the Optotrak), $\mathbf{M}_{1}$ is the transformation from 3-D image space to 


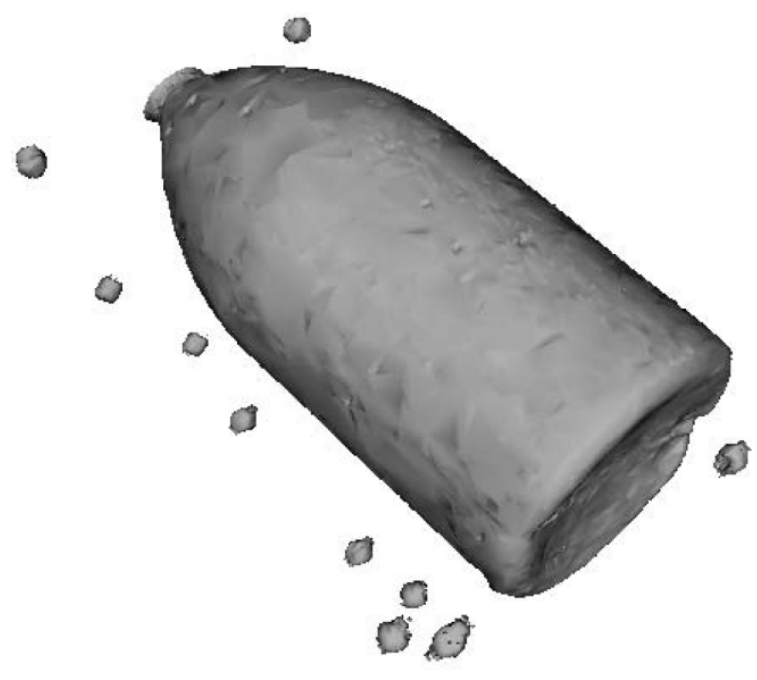

(a)

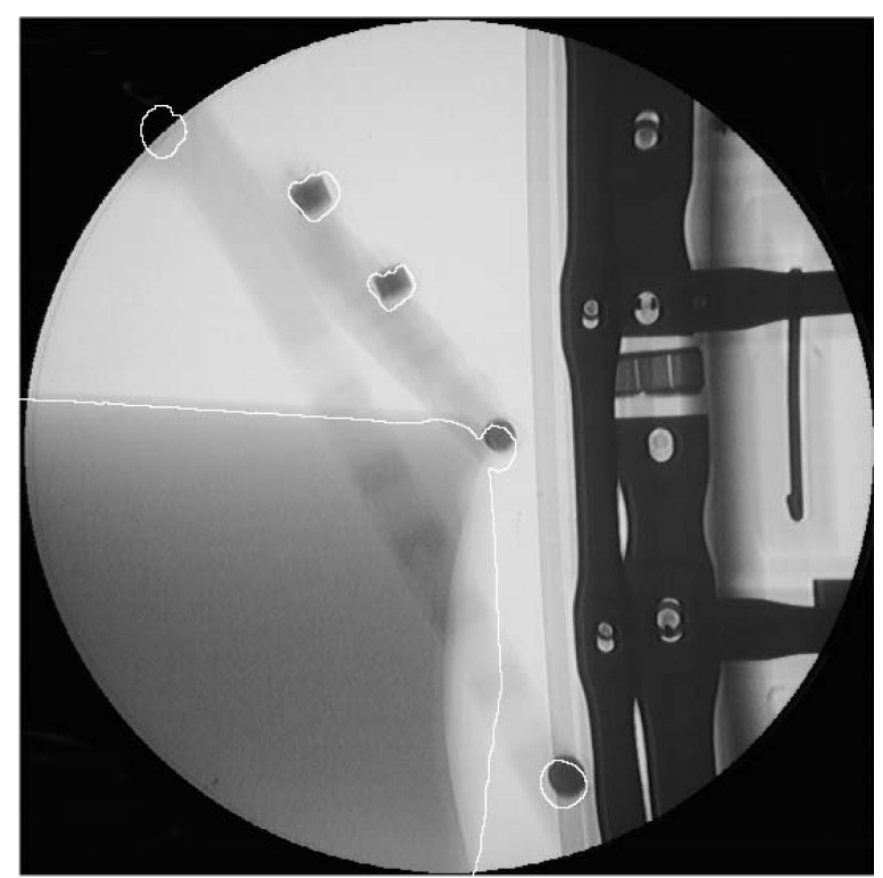

(b)

Fig. 4. (a) Rendering derived from the MR volume scan of the calibration object. The bottle used for loading is also displayed. (b) X-ray view of the calibration object used for the point-based validation experiment. The contour-traced MR MIP generated using the registration matrix is also shown. $\mathrm{FOV}=23 \mathrm{~cm}$.

scanner space (determined from image header information), and $\mathbf{F}_{I}$ is the matrix of marker locations in 3-D image space. Equation (3) is solved to compute $\mathbf{M}_{2}$, the transformation from scanner space to $\mathrm{X}$-ray table space. Matrix $\mathbf{M}_{2}$ is particular for the calibration object but the overall transformation from 3-D image space to X-ray table space must also include a variable translation parallel to the axis of motion of the sliding table top. The unit translation $\mathbf{T}$ is calculated by moving the calibration object along the sliding table top parallel to its axis of motion and marking the position of physical markers using the pointing device and the Optotrak. The magnitude of the translation $\lambda$ is determined from the tracking of the sliding table top provided by the MR scanner software.

\section{E. Generation of the Overall Registration Matrix}

Once the calibration steps have been carried out it is then possible to generate a 3-D to 2-D registration matrix for any MR scans and tracked X-ray images. The matrices $\mathbf{M}_{2}, \mathbf{T}$, and $\mathbf{P}$ are determined from the calibration, whereas $\mathbf{M}_{1}, \mathbf{M}_{3}$, and the parameter $\lambda$ are specific to the MR acquisition and the tracked $\mathrm{X}$-ray images. Furthermore, since the radiographer is allowed to arbitrarily rotate the X-ray image for correct anatomical viewing, the 2-D rotation matrix $\mathbf{R}$ needs to be determined by marking corresponding points in 2-D images acquired pre- and post-rotation. The overall registration matrix is then given by

$$
\mathbf{W}=\mathbf{R P M}_{3} \lambda \mathbf{T M}_{2} \mathbf{M}_{1} .
$$

\section{F. Validation With Point-Based Test Object}

The first validation experiment was carried out using the calibration object described above as a phantom.

Using the MR imaging markers, the phantom was placed in the head coil on the table top docked to the MR scanner, and imaged using a T1-weighted volume MR scan $(256 \times 256$ matrix, 200 slices, resolution $=1.05 \mathrm{~mm} \times 1.05 \mathrm{~mm} \times 1.2 \mathrm{~mm}$, $\mathrm{TR}=14.0 \mathrm{~ms}, \mathrm{TE}=6.0 \mathrm{~ms}$, flip angle $=15^{\circ}$ ). The table top was then docked to the X-ray system, and five pairs of tracked X-ray images were acquired. Each pair of images simulated the approximately orthogonal views typically acquired during an intervention. The registration matrix was calculated for each image and this was used to generate maximum intensity projections (MIPs) through the MR volume. Note the registration matrix includes the X-ray perspective geometry, so these MIPs have the same perspective as the X-ray images, unlike MIPs calculated by the scanner console that are parallel projections. Fig. 4(b) shows one of the X-ray views with the MR MIP overlaid. The 2-D registration error was quantified by marking the location of the imaging markers in both the X-ray images and the MIPs. In total 35 markers where located in 2-D. The 3-D error was found by finding the position of the markers from the biplane MIPs using the epipolar constraint [10], [11] and by manual marking in the MR volume scan. In total 17 markers where located in 3-D.

\section{G. Validation With Anthropomorphic Phantom}

The second set of validation experiments were carried out with an anthropomorphic vascular phantom. This incorporates a silicone model of one half of the circle of Willis (supplied by Professor D. Rufenacht and Dr. K. Tokunaga of the Division of Neuro-Radiology, Geneva University Hospital, Switzerland). The model is normally used for the training of neuroradiologists to carry out cerebral aneurysm coiling and was constructed using a post-mortem pathological brain specimen. The model has one in-flow vessel (internal carotid artery) and three outflow vessels (anterior, middle, and posterior cerebral arteries). The model was mounted inside an acrylic box with three-way valves connecting the vessels to the outside of the box. The box was filled with gelatin to simulate surrounding brain tissue and to give realistic X-ray scatter and attenuation. It was connected to a pulsatile flow pump that circulated saline with an average flow of approximately $450 \mathrm{ml}$ per minute. It is worth noting that 
the vessels in this phantom are considerably smaller (largest diameter: $5 \mathrm{~mm}$ ) than the vessels in the chest that are of interest in the clinical validation described below (aorta, pulmonary arteries, etc.), which makes any errors visually more apparent. Nine MR imaging markers were placed on the vascular phantom to assess the registration error.

The phantom was imaged using a phase contrast MR sequence $(256 \times 256$ matrix, 110 slices, resolution = $0.86 \mathrm{~mm} \times 0.86 \mathrm{~mm} \times 0.86 \mathrm{~mm}, \mathrm{TR}=9.2 \mathrm{~ms}, \mathrm{TE}=4.3 \mathrm{~ms}$, flip angle $=20^{\circ}$, maximum velocity encoding $=100 \mathrm{~cm} / \mathrm{s}$ ) and a T1-weighted volume MR sequence $(512 \times 512$ matrix, 190 slices, resolution $=0.43 \mathrm{~mm} \times 0.43 \mathrm{~mm} \times 0.8 \mathrm{~mm}$, $\mathrm{TR}=7.3 \mathrm{~ms}, \mathrm{TE}=2.0 \mathrm{~ms}$, and flip angle $\left.=20^{\circ}\right)$. It was then transferred to the $\mathrm{X}$-ray system and tracked dynamic images were acquired from two oblique views separated by approximately 30 degrees. The imaging was carried out during the injection of iodine contrast medium.

The registration transformations described in Section II-E provide the relative orientation of the two X-ray views. Using the registration matrices and the epipolar constraint it is possible to semi-automatically track vessels from the two X-ray views [12]-[17]. This provides a 2-D centerline representation for each X-ray view, together with a single 3-D centerline representation for each pair of views. This was carried out for the internal carotid artery segment of the vascular phantom.

The registration matrices were used to generate MR MIPs corresponding to the X-ray projections. The 2-D centerlines of the vessels in these MIPs, and the corresponding 3-D centerline could be calculated using the same algorithm used for the X-ray images.

A 2-D registration error was also calculated from the location of the imaging markers in the X-ray images and the MR MIPS. A total of 12 markers were visible in the pair of X-ray images.

The target registration error (TRE) was quantified in 2-D and 3 -D using the centerline representation above. For each point in the MR centerline, the closest corresponding point in the registered X-ray centerline was determined. The distances between these closest points was determined in 2-D for the 2-D centerlines, giving a 2-D TRE, and in 3-D for the 3-D centerlines, giving a 3-D TRE. It is important to assess both these errors, as the 2-D TRE relates to the accuracy of any overlay of MR images, and the 3-D TRE relates to the error in locating structures in 3-D.

\section{H. Clinical Validation}

The patients investigated all gave informed consent for the use of their medical records in this research, as part of a local research ethics committee approved protocol. This protocol required that the cardiac catheterization procedures were guided using the X-ray and MRI images alone, not with the experimental software described in this paper.

The system was calibrated prior to each patient investigation using the technique described in Sections II-C and D with IRED markers fixed to the X-ray table and c-arm. However, there was no need to fix any markers to the patients. Images from two patients undergoing cardiac catheterization at the Guy's XMR facility were utilized. During the MR part of the procedure, MR scans of the heart and the great vessel were acquired using an SSFP three-dimensional multiphase sequence (typically three phases, $256 \times 256$ matrix, 128 slices, resolution $=$ $1.13 \mathrm{~mm} \times 1.13 \mathrm{~mm} \times 1.0 \mathrm{~mm}, \mathrm{TR}=3.1 \mathrm{~ms}, \mathrm{TE}=1.6 \mathrm{~ms}$, and flip angle $=45^{\circ}$ ). During the X-ray part of the procedure, a frame grabber was used to acquire tracked X-ray images. The cardiologists carrying out the intervention could move the X-ray $\mathrm{C}$-arm or table in any way they wished, but all images used in this analysis were acquired with the minimum image intensifier magnification, as used in the calibration. Registration matrices were calculated for each of the tracked views. In order to overcome the problems of cardiac and respiratory motion, image acquisition was ventilator-controlled and only end-diastolic MR and X-ray images were used.

1) Case 1. Pulmonary Vascular Resistance Study: Patient 1, male aged 16, required the invasive measurement of pressure in the pulmonary system in order to quantify pulmonary vascular resistance. Registration of the X-ray and MR images was designed to enable the position of the pressure catheter under X-ray to be linked in 3-D to MR flow measurements.

2) Case 2. Cardiac RF Ablation to Treat Arrhythmia: Patient 2, male aged 15, had an intermittent ventricular tachycardia that was to be treated by electrophysiology study and RF ablation under X-ray guidance. The catheters used for these procedures are not MR compatible, as they are comprised of long electrical conductors. Registration of the X-ray and MR images was designed to enable the position of the electrophysiology measurement catheters to be related to the MR-derived cardiac anatomy. For this patient, tagged MR sequences $(256 \times 256$ matrix, 59 phases, resolution $=1.33 \mathrm{~mm} \times 1.33 \mathrm{~mm} \times 8.0 \mathrm{~mm}, \mathrm{TR}=11.0 \mathrm{~ms}$, $\mathrm{TE}=3.5 \mathrm{~ms}$, flip angle $=13^{\circ}$, and tag spacing $=8 \mathrm{~mm}$ ) were also acquired from which the myocardial motion was quantified using a nonrigid registration technique [18], [19]. The registration enabled us to relate the position of the measured electrophysiology data to the cardiac motion.

\section{RESULTS}

\section{A. Calibration}

Fig. 5 shows how the four intrinsic perspective parameters, $\mathbf{I}=\left(c_{s}, l_{s}, k_{1}, k_{2}\right)$, vary with angle of rotation of the X-ray $\mathrm{C}$-arm from $0^{\circ}$ (AP) to $90^{\circ}$ (lateral). The position of the imaging plane normal which passes through the X-ray source is defined by $c_{s}$ and $l_{s}$, whilse $k_{1}$ and $k_{2}$ give the ratios of the X-ray pixel sizes to the focal length [25]. The variation is sufficient to show that calibration from a single view is inappropriate. We, therefore, calculate an average perspective projection, as described above, for all subsequent experiments. The robustness of this average projection was assessed using a leave-one-out experiment. The 2-D root-mean-square error between the position of markers located in the X-ray images and the position of markers calculated using the perspective projection matrix was found to be $2.0 \mathrm{~mm}(n=48)$. This calibration technique was applied to the phantom and clinical studies.

\section{B. Validation With Point-Based Test Object}

2-D TRE calculated from the location of the markers in the $\mathrm{X}$-ray images and the MR MIPs generated using the registration 


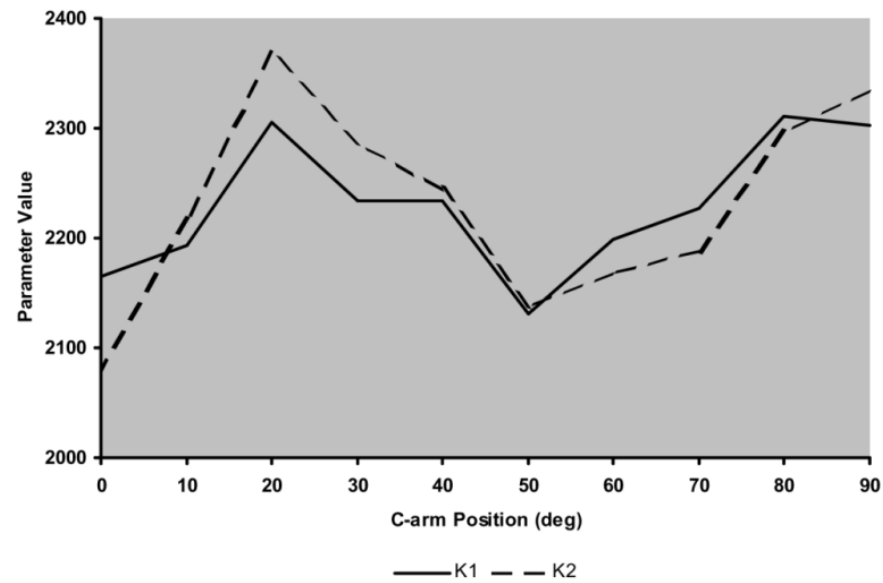

(a)

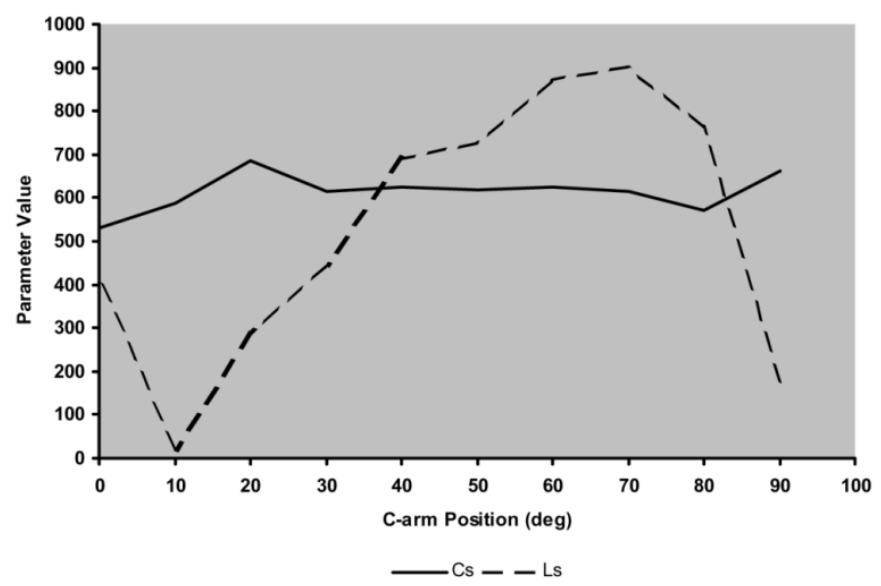

(b)

Fig. 5. Results from conventional perspective projection calibration experiment showing the variation of perspective projection parameters with the orientation of the X-ray system C-arm, (a) for parameters $k 1$ and $k 2$, and (b) for parameter cs and $1 \mathrm{~s}$.

matrices was found to be $4.2 \mathrm{~mm}(n=35)$. The 3-D TRE calculated from the location of markers MR volume and the pairs of X-ray views was found to be $4.6 \mathrm{~mm}(n=17)$.

\section{Validation With Anthropomorphic Phantom}

Fig. 6(a) shows one of the two X-ray views of the vascular phantom. Fig. 6(b) is the MR MIP corresponding to this view generated using the registration matrix. Fig. 7(a) shows the combination of the X-ray image and the MR MIP. Fig. 7(b) shows the same X-ray view with the MR vessel centerline overlaid. The 2-D TRE for the target vessel centerline was found to be 2.7 $\mathrm{mm}(n=255)$ for the first view and $2.4 \mathrm{~mm}(n=242)$ for the second view. The 3-D TRE was found to be $5.1 \mathrm{~mm}(n=233)$ computed from both views. The 2-D TRE computed using the imaging markers was $3.6 \mathrm{~mm}(n=12)$. The 2-D TRE calculated using the markers was approximately $1 \mathrm{~mm}$ greater than that using the vessel centerlines. This was thought to be partly due to the fact that the markers were distributed around the periphery of the vascular phantom whereas the target vessel was centrally placed. X-ray geometric distortion would, therefore, affect the accuracy of locating the marker positions to a greater extent than locating the vessel centerline positions.

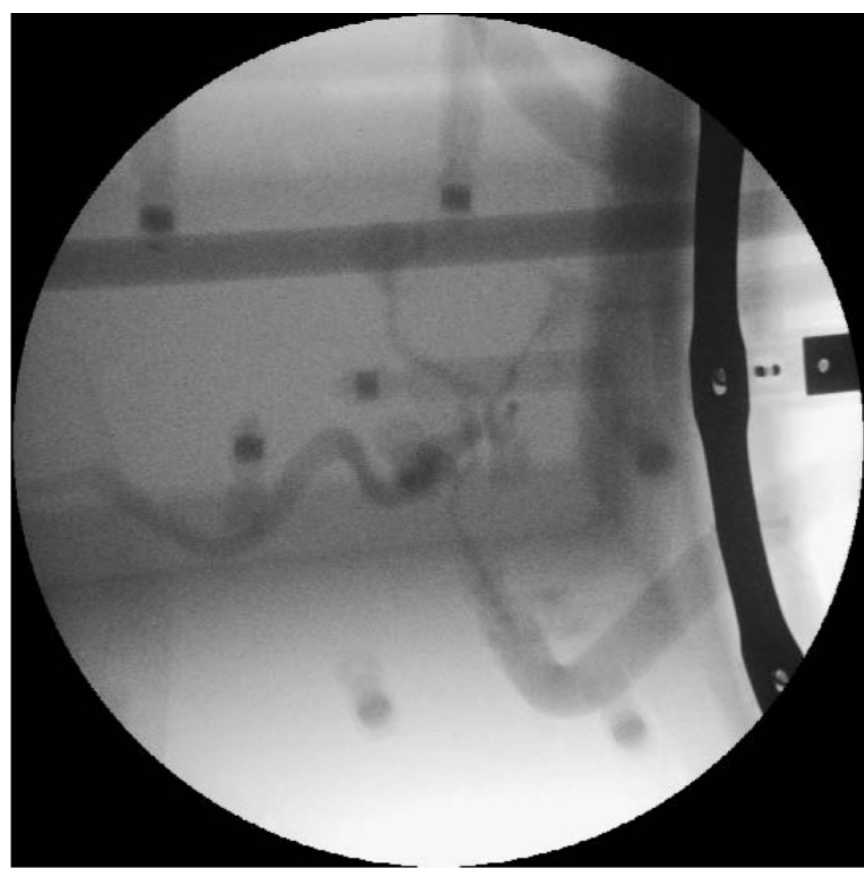

(a)

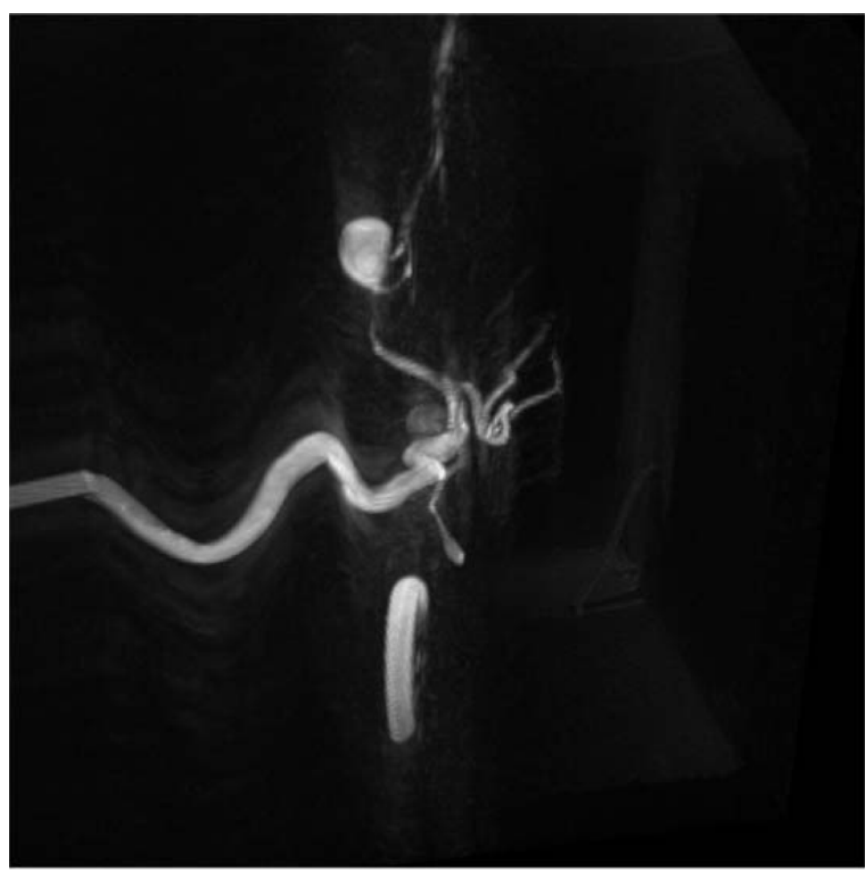

(b)

Fig. 6. (a) One of the two X-ray views of the vascular phantom. (b) Corresponding MR MIP generated using the registration matrix.

\section{Clinical Validation}

1) Case 1: Fig. 8 shows the bi-plane $X$-ray images acquired while a catheter was positioned in the right pulmonary artery of this patient. Also shown are two surface renderings of the heart incorporating the 3-D reconstruction of the catheter obtained from the registered bi-plane $\mathrm{X}$-ray views. The surfaces were generated using the marching cubes algorithm after segmenting the 3-D MR heart volume using the Analyze software package (Mayo Clinic, Rochester, MN). 


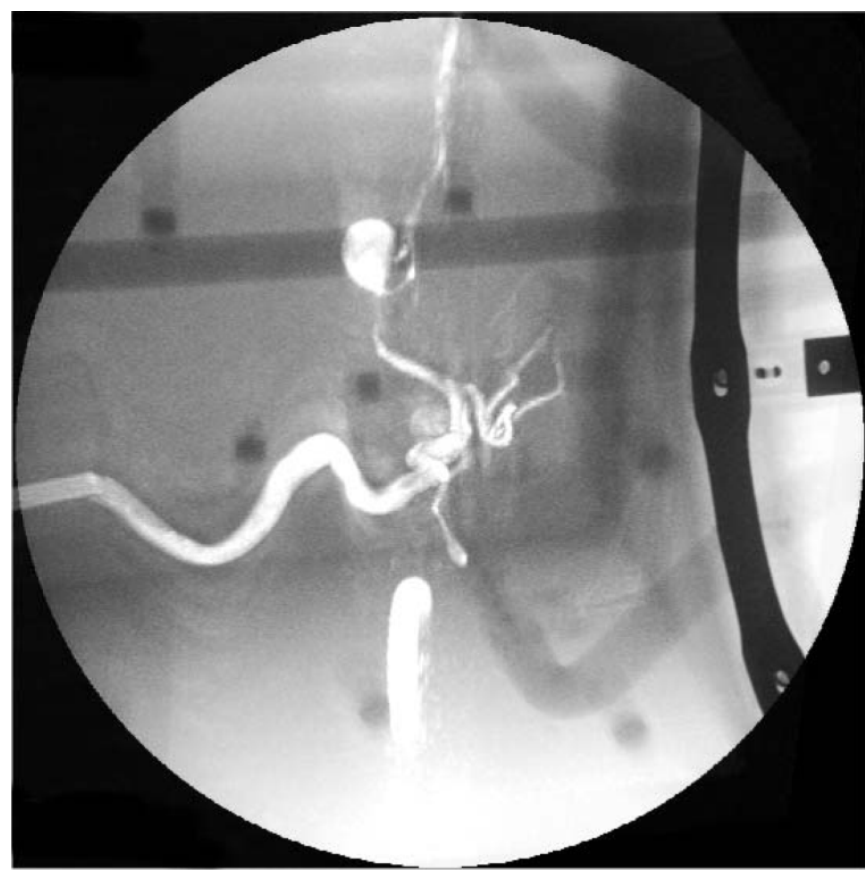

(a)

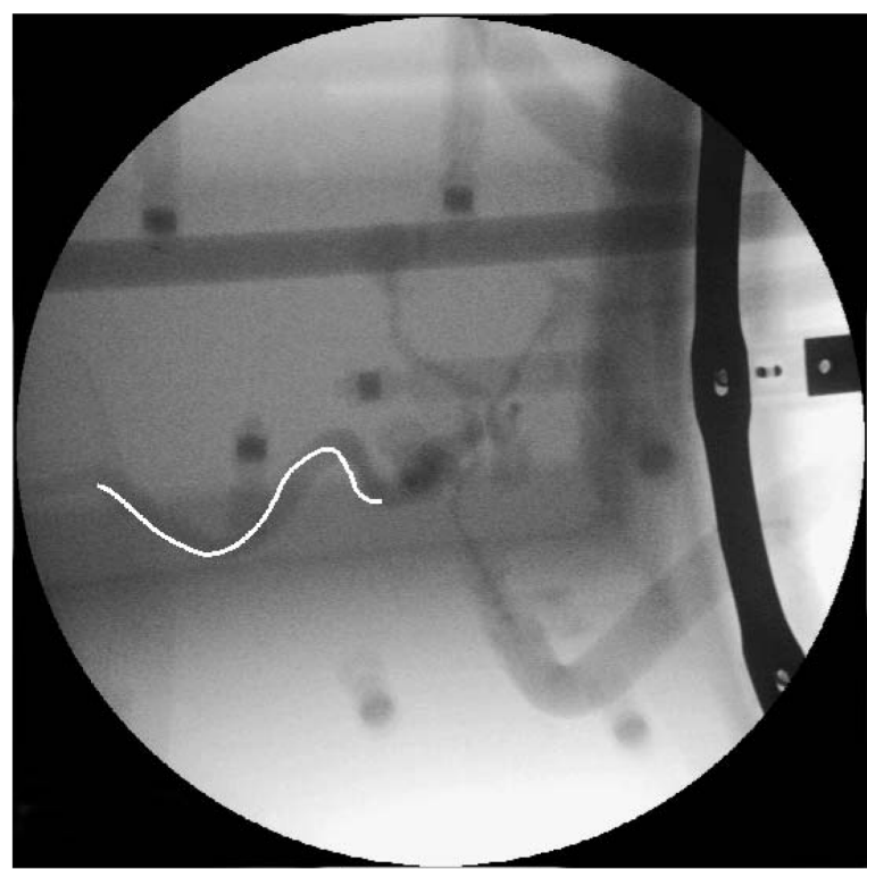

(b)

Fig. 7. (a) Combined X-ray image and MR MIP of vascular phantom from Fig. 6. (b) X-ray image with the 2-D centerline determined from the MR MIP.

2) Case 2: Fig. 9 shows the bi-place $\mathrm{X}$-ray views acquired during the electrophysiology study for this patient. Four catheters can be seen in place. Three of these are measurement catheters with several electrodes along their lengths, and the other is a basket catheter with 67 electrodes distributed approximately over a spherical surface. Fig. 10 shows the MR-derived surface rendering of the right side of this patient's heart. Added to this are the three measurement catheters reconstructed using the registered bi-plane X-ray views and the basket catheter shown as a sphere whose center and diameter were found from the registered views. Fig. 11 is a rendering that shows
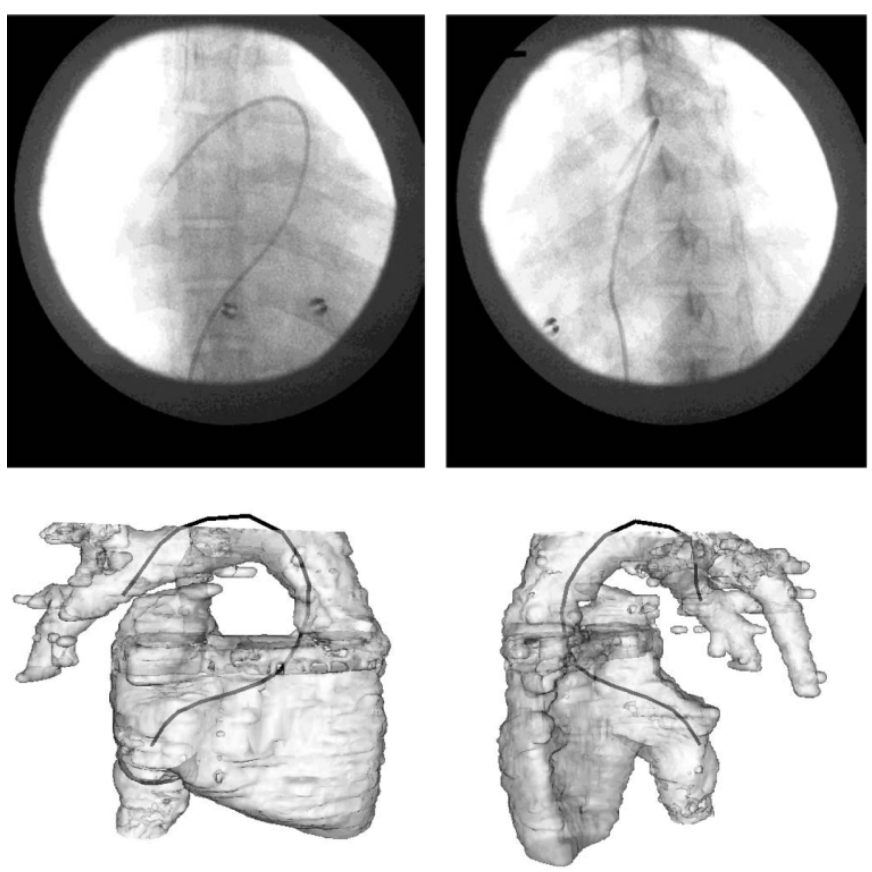

Fig. 8. Case 1. Bi-plane X-ray views of catheter in the right pulmonary artery and two views of the surface rendering of the right side of the heart derived from the MR images and the catheter reconstructed using the registered bi-plane $\mathrm{X}$-rays views.

the electrode basket, a slice of the tagged MR image and the myocardial motion vectors for the right ventricle. The motion vectors were derived from the dynamic tagged images using nonrigid registration.

\section{DISCUSSION AND CONCLUSION}

Recently introduced XMR systems, in which MRI and X-ray imaging are available in the same room, allow endovascular procedures to be carried out under a combination of MR and X-ray guidance. We have described our novel approach for the registration of these imaging modalities to provide an integrated representation of the patient for use during guidance. Our technique determines the relative positions of the X-ray set, patient and MR scanner using a combination of IREDs tracked by an Optotrak, and the MR scanner's own table tracking system. Having carried out an initial calibration stage, this tracking information enables us to generate a projection from an MR image to match the current X-ray view, and also to reconstruct the 3-D position of a point or line from a pair of X-ray views, and transfer this 3-D structure into MRI coordinates. The accuracy of our X-ray perspective projection matrix calculation technique was found to be $2.0 \mathrm{~mm}(n=48)$. Results from a point-based phantom validation experiment showed a 2-D TRE of $4.2 \mathrm{~mm}(n=35)$ and a 3-D TRE of $4.6 \mathrm{~mm}(n=17)$. Validation of our registration technique with an anthropomorphic vascular phantom produced 2-D TREs of 2.4 and $2.7 \mathrm{~mm}(n=242, n=255$, respectively) for 2-D vessel centerlines, and a 3-D TRE of $5.1 \mathrm{~mm}(n=233)$ for a 3-D vessel centerline. A 2-D TRE of $3.6 \mathrm{~mm}(n=12)$ was also found for the fiducial markers attached to the vascular phantom. The results of our phantom studies were within the accuracy required to register X-ray and MR images of the heart and the great vessels. 


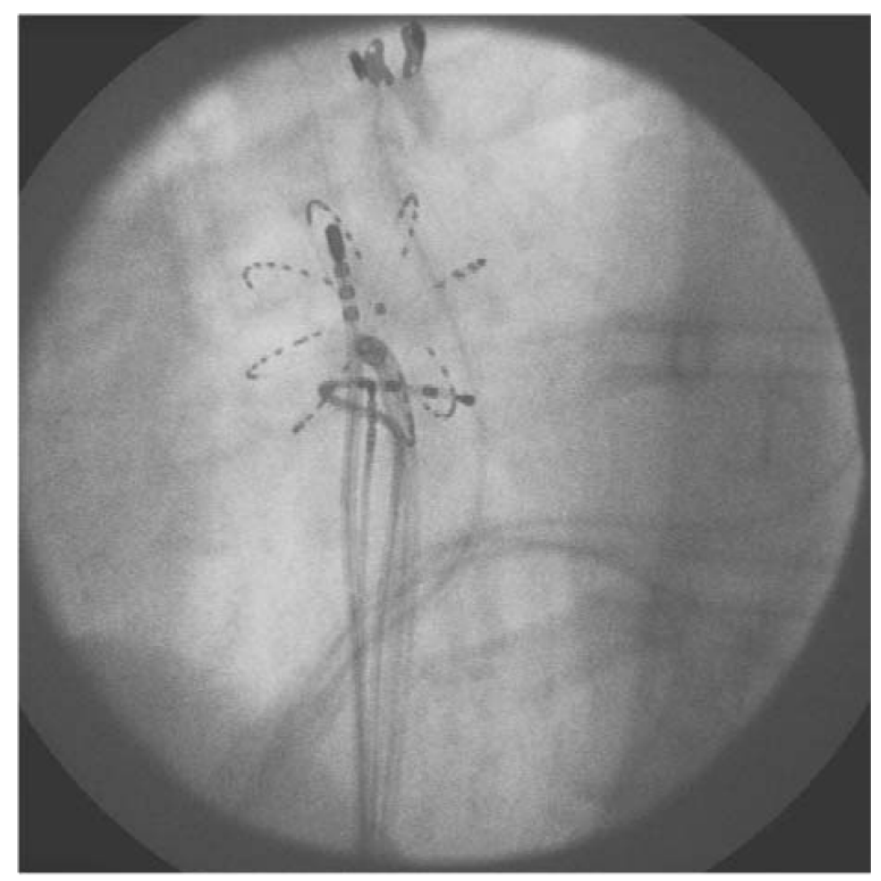

(a)

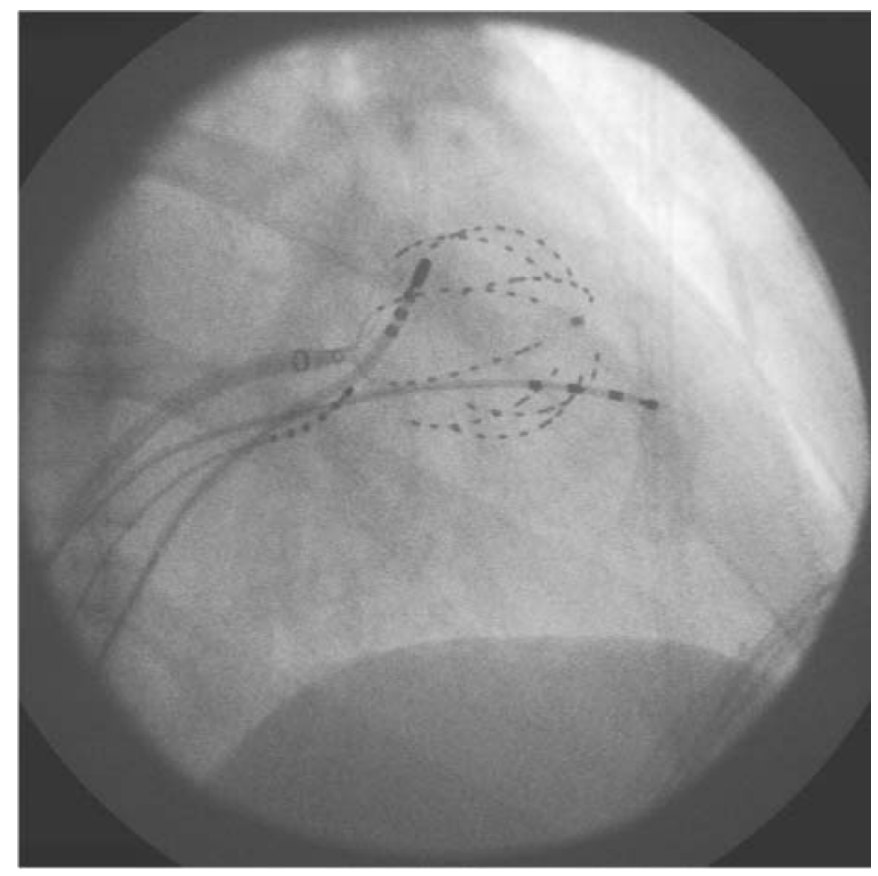

(b)

Fig. 9. Case 2. Bi-plane X-ray views showing three electrical measurement catheters and one electrode basket catheter in the right ventricle.

Our registration technique was subsequently applied to images acquired during two clinical studies. The technique was used to overlay registered projections derived from MR volume images onto X-ray images of the heart and great vessels. It was also used to reconstruct the 3-D position of catheters seen using $\mathrm{X}$-ray imaging within previously acquired MR volume images. The accuracy of these representations was assessed to be of the same order as found in our phantom studies.

For the first clinical case, part of the procedure was carried out under MR guidance and part under X-ray guidance. Blood flow measurements were made using MR imaging and pressure measurements were carried out under X-ray guidance. Our registration technique allowed the position of these measurements to be related in the same coordinate system. This information could subsequently be used for haemodynamics assessment of the patient.

The second clinical case was a patient undergoing electrophysiology study and RF ablation for a ventricular tachycardia. The lack of visualization of the heart and great vessels during $\mathrm{X}$-ray-guided cardiac catheterization is a real disadvantage especially during interventional procedures such as cardiac RF ablation. This leads to prolongation of the procedure time, increased X-ray radiation dose and is often the main cause for unsuccessful outcome and the need for repeat cardiac catheterization. Therefore, especially for RF ablation procedures, much research has been carried out into incorporating some anatomical information to aid interventional guidance. This includes the use of transoesophageal and intracardiac echocardiography, and CARTO ${ }^{1}$ and ESI $^{2}$ electroanatomical mapping systems [20]. There is a relationship between the electrical activation and motion of the myocardium. For the second clinical case we were able to measure the motion by processing tagged MR images and our registration method allowed us to relate the location of the measurement electrodes positioned under X-ray guidance to the MR-derived motion. In the future this opens up a new avenue of research and, as well as providing extra information for the cardiologist during the ablation procedure, it has the potential of replacing part of the invasive X-ray-guided cardiac electrophysiology study with noninvasive MR-guidance.

Our system is not yet real-time, as there are delays in the transfer and pre-processing of the MR images, and the results shown in this paper were generated after the end of the procedures. The most significant bottleneck is the need to segment the 3-D cardiac MR volumes so that the MR overlays show only the anatomy of interest. However, once the segmented data is available, registered images can be generated in less than $15 \mathrm{~s}$, which is sufficient for the integrated images to be generated during a procedure, even if they are not generated sufficiently fast to be updated while the cardiologist is manipulating a device. In ongoing work, we are upgrading the system to provide near real-time registered images available to the cardiologist during the procedure.

Our phantom results show that the potential accuracy of the system is approximately $4 \mathrm{~mm}$ at present. Patient motion during a procedure will reduce this accuracy. The patients investigated in our center all have a general anaesthetic and paralysis. This reduces the likelihood of the patient moving substantially on the table, and also enables us to overcome respiratory motion by acquiring the images with a ventilator-controlled end-expiration image acquisition. Visual inspection of the results from the patient study show that the accuracy is of the same order of magnitude as the accuracy of the phantom studies. The accuracy could be further improved by better synchronization with the cardiac cycle, and refinement of the registration transformation using an intensity-based registration algorithm. Inten-

\footnotetext{
${ }^{1}$ www.biosensewebster.com

${ }^{2}$ www.endocardial.com
} 


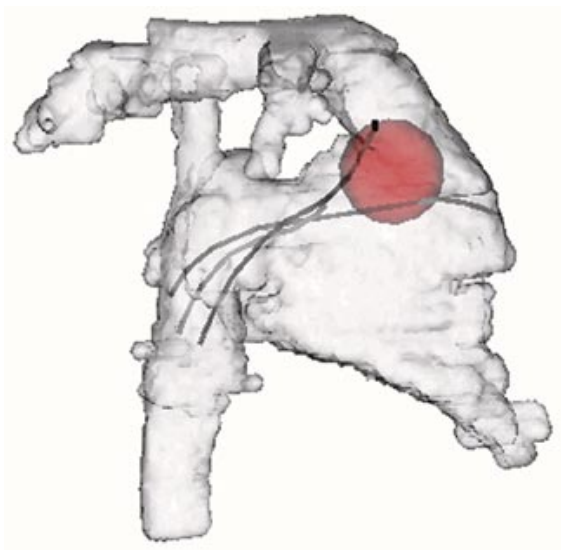

(a)

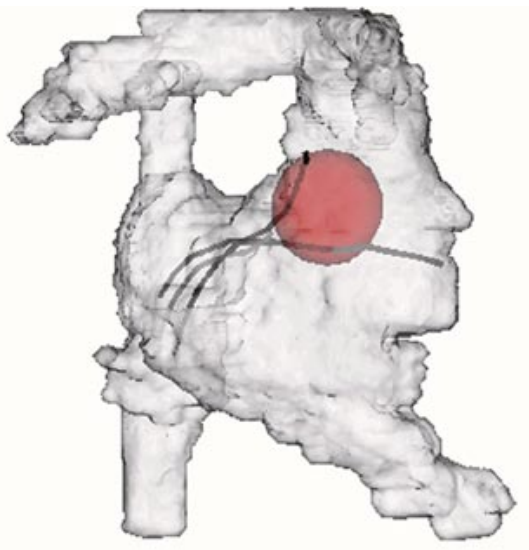

(b)

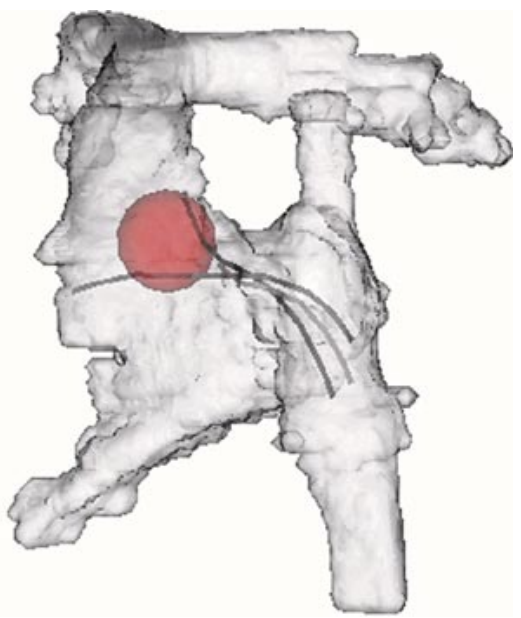

(c)

Fig. 10. Case 2. Surface rendering of the right side of the heart derived from the MR images incorporating the three reconstructed catheters and the basket catheter shown as a sphere. (a) AP, (b) Oblique, and (c) PA views.

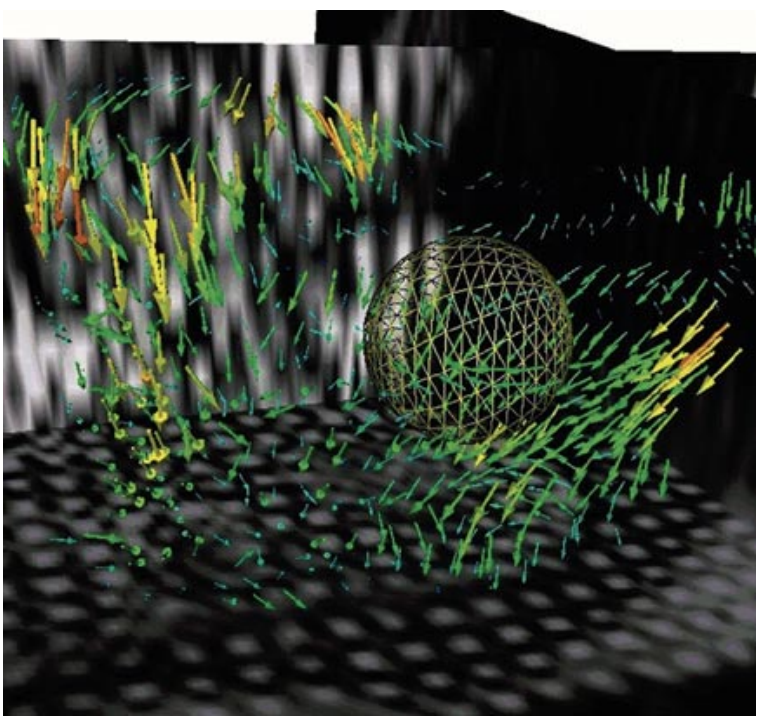

Fig. 11. Case 2. The electrode basket, tagged MR image slices, and derived motion vectors all displayed in the same coordinate system.

sity-based 2-D-3-D registration algorithms calculate a registration matrix by iteratively generating a digitally reconstructed radiograph (DRR) from a volume data set and comparing this with a radiographic image until a best match is obtained. Such algorithms have been applied to the registration of computed tomography data to radiographs of skull [21], femur [22], and spine phantoms [23]. Clinical applications have included patients undergoing endovascular treatment for abdominal aortic aneurysm [24] and endovascular treatment of cerebral aneurysms [25]. These techniques have also been used for the positioning of patients undergoing radiotherapy [26], [27]. The performance of different similarity measures has been investigated to determine the best match between DRRs and radiographs [23], [25], [27]-[29]. We are currently extending our technique by using the optical tracking solution as a starting estimate for an intensity-based registration approach, which we expect will improve our accuracy.

\section{ACKNOWLEDGMENT}

The authors acknowlege that his work would not have been possible without the dedication of the multidisciplinary team working at their XMR facility. The authors would like to acknowledge that the scanner was purchased using grants from the UK JREI, Philips Medical Systems, and the Charitable Foundation of Guy's \& St Thomas' Hospitals.

\section{REFERENCES}

[1] R. Fahrig, K. Butts, J. A. Rowlands, R. Saunders, J. Stanton, G. M. Stevens, B. L. Daniel, Z. Wen, D. L. Ergun, and N. J. Pelc, "A truly hybrid interventional MR/X-ray system: Feasibility demonstration," $J$. Magn. Reson. Imag., vol. 13, no. 2, pp. 294-300, 2001.

[2] R. Fahrig, K. Butts, Z. F. Wen, R. Saunders, S. T. Kee, D. Y. Sze, B. L. Daniel, F. Laerum, and N. J. Pelc, "Truly hybrid interventional MR/X-ray system: Investigation of in vivo applications," Acad. Radiol., vol. 8, no. 12, pp. 1200-1207, 2001.

[3] G. Adam, J. Neuerburg, A. Buecker, A. Glowinski, D. Vorwerk, A. Stargardt, J. J. VanVaals, and R. W. Gunther, "Interventional magnetic resonance - Initial clinical experience with a 1.5-tesla magnetic resonance system combined with c-arm fluoroscopy," Investigat. Radiol., vol. 32, no. 4, pp. 191-197, 1997.

[4] A. Buecker, J. M. Neuerburg, G. B. Adam, A. Glowinski, T. Schaeffter, V. Rasche, J. J. van Vaals, A. Molgaard-Nielsen, and R. W. Guenther, "Real-time MR fluoroscopy for MR-guided iliac artery stent placement," J. Magn. Reson. Imag., vol. 12, no. 4, pp. 616-22, 2000.

[5] A. Buecker, E. Spuentrup, R. Grabitz, F. Freudenthal, E. G. Muehler, T. Schaeffter, J. J. van Vaals, and R. W. Gunther, "Magnetic resonanceguided placement of atrial septal closure device in animal model of patent foramen ovale," Circulation, vol. 106, no. 4, pp. 511-515, 2002.

[6] A. Buecker, G. B. Adam, J. M. Neuerburg, S. Kinzel, A. Glowinski, T. Schaeffter, V. Rasche, J. J. van Vaals, and R. W. Guenther, "Simultaneous real-time visualization of the catheter tip and vascular anatomy for MR-guided PTA of iliac arteries in an animal model," J. Magn. Reson. Imag., vol. 16, no. 2, pp. 201-208, 2002.

[7] T. J. Vogl, J. O. Balzer, M. G. Mack, G. Bett, and A. Oppelt, "Hybrid MR interventional imaging system: Combined MR and angiography suites with single interactive table. Feasibility study in vascular liver tumor procedures," Eur. Radiol., vol. 12, no. 6, pp. 1394-1400, 2002.

[8] C. R. Maurer Jr., J. M. Fitzpatrick, M. Y. Wang, R. L. Galloway Jr., R. J. Maciunas, and G. S. Allen, "Registration of head volume images using implantable fiducial markers," IEEE Trans. Med. Imag., vol. 16, pp. 447-462, Oct. 1997. 
[9] P. J. Edwards, A. P. King, C. R. Maurer Jr., D. A. DeCunha, D. J. Hawkes, D. L. G. Hill, R. P. Gaston, M. J. Clarkson, M. R. Pike, M. R. Fenlon, S. Chandra, A. J. Strong, C. L. Chandler, and M. J. Gleeson, "Design and evaluation of a system for microscope-assisted guided interventions (MAGI)," IEEE Trans. Med. Imag., vol. 19, pp. 1082-1093, Feb. 2000.

[10] D. J. Hawkes, A. C. F. Colchester, and C. Mol, "The accurate 3D reconstruction of the geometric configuration of vascular trees from X-ray recordings," in Physics and Engineering of Medical Imaging, Guzzardi, Ed. The Hague, The Netherlands: Martinus Nijhoff, 1985.

[11] K. R. Hoffman, A. Sen, L. Lan, C. E. Metz, K. Chua, B. B. Williams, J. Esthappen, M. Fiebich, M. Mazzucco, and K. Doi, "Determination of 3-D vessel trees from biplane images from coronary angiography," Comput. Assist. Radiol. Surg., pp. 162-165, 1997.

[12] A. M. Seifalian, D. J. Hawkes, A. C. F. Colchester, and K. E. F. Hobbs, "A new algorithm for deriving pulsatile blood-flow waveforms tested using simulated dynamic angiographic data," Neuroradiology, vol. 31, pp. 263-269, 1989

[13] D. J. Hawkes, A. M. Seifalian, A. C. F. Colchester, N. Iqbal, C. R. Hardingham, C. F. Bladin, and K. E. F. Hobbs, "Validation of volume blood-flow measurements using 3-dimensional distance-concentration functions derived from digital X-ray angiograms," Investigat. Radiol., vol. 29, pp. 434-442, 1994.

[14] A. M. Seifalian, D. J. Hawkes, C. F. Bladin, A. C. F. Colchester, and K. E. F. Hobbs, "Blood flow measurements using 3D distance-concentration functions derived from digital X-ray angiograms," Cardiovasc. Imag., pp. 425-442, 1996

[15] K. S. Rhode, T. Lambrou, D. J. Hawkes, G. Hamilton, and A. M. Seifalian, "Validation of an optical flow algorithm to measure blood flow waveforms in arteries using dynamic digital X-ray images," Proc. SPIE (Med. Imag.), vol. 3979, pp. 1414-1425, 2000.

[16] K. S. Rhode, G. Ennew, T. Lambrou, D. J. Hawkes, and A. M. Seifalian, "In-vitro validation of a novel model-based approach to the measurement of arterial blood flow waveforms from dynamic digital X-ray images," in Lecture Notes in Computer Science, Springer. Berlin, Germany: Springer-Verlag, 2001, vol. 2208, Proc. Medical Image Computing Computer-Assisted Intervention - MICCAI, pp. 291-300.

[17] K. S. Rhode, T. Lambrou, A. M. Seifalian, and D. J. Hawkes, "In-vitro validation of a novel model-based approach to the measurement of arterial blood flow waveforms from dynamic digital X-ray images," Proc. SPIE (Med. Imag.), vol. 4683, pp. 286-296, 2002.

[18] D. Rueckert, L. I. Sonoda, C. Hayes, D. L. G. Hill, M. O. Leach, and D. J. Hawkes, "Non-rigid registration using free-form deformations: Application to breast MR images," IEEE Trans. Med. Imag., vol. 18, pp. 712-721, Aug. 1999.
[19] R. Chandrashekara, R. H. Mohiaddin, and D. Rueckert, "Analysis of myocardial motion in tagged MR images using nonrigid image registration," Proc. SPIE (Med. Imag.), vol. 4684, pp. 1168-1179, 2002.

[20] J. L. R. M. Smeets, S. A. Ben-Haim, L.-M. Rodriguez, C. Timmermans, and H. J. J. Wellens, "New method for nonfluoroscopic endocardial mapping in humans. Accuracy assessment and first clinical results," Circulation, vol. 97, pp. 2426-2432, 1998.

[21] L. Lemieux, R. Jagoe, D. R. Fish, N. D. Kitchen, and D. G. T. Thomas, "A patient-to-computed-tomography image registration method based on digitally reconstructed radiographs," Med. Phys., vol. 21, no. 11, pp. 1749-1760, 1994.

[22] L. M. G. Brown and T. E. Boult, "Registration of planar film radiographs with computed tomography," in Proc. MMBIA, 1996, pp. 42-51.

[23] G. P. Penney, J. Weese, J. A. Little, P. Desmedt, D. L. G. Hill, and D. J. Hawkes, "A comparison of similarity measures for use in 2-D-3-D medical image registration," IEEE Trans. Med. Imag., vol. 17, pp. 586-595, 1998.

[24] G. P. Penney, P. G. Batchelor, D. L. G. Hill, D. J. Hawkes, and J. Weese, "Validation of a two- to three-dimensional registration algorithm for aligning preoperative CT images and intraoperative fluoroscopy images," Med. Phys., vol. 28, no. 6, pp. 1024-1032, 2001.

[25] J. H. Hipwell, G. P. Penney, T. C. S. Cox, J. V. Byrne, and D. J. Hawkes, "2D-3D intensity based registration of DSA and MRA - A comparison of similarity measures," Lecture Notes in Computer Science, vol. 2489, pp. 501-508, 2002.

[26] K. G. A. Gilhuijs, P. J. H. van de Ven, and M. van Herk, "Automatic three-dimensional inspection of patient setup in radiation therapy using portal images, simulator images, and computed tomography data," Med. Phys., vol. 23, no. 3, pp. 389-399, 1996.

[27] N. Dekker, L. S. Ploeger, and M. van Herk, "Evaluation of cost functions for gray value matching of two-dimensional images in radiotherapy," Medical Physics, vol. 30, no. 5, pp. 778-784, 2003.

[28] T. Rohlfing and C. R. Maurer Jr., "A novel image similarity measure for registration of 3D MR images and X-ray projection images," in Proc. Medical Image Computing Computer-Assisted Intervention - MICCAI, vol. 2489, 2002, pp. 469-476.

[29] T. Rohlfing, D. B. Russakoff, M. J. Murphy, and C. R. Maurer Jr., "An intensity-based registration algorithm for probablistic images and its application for 2-D to 3-D image registration," Proc. SPIE (Med. Imag.), vol. 4684, pp. 581-591, 2002. 